\title{
Evolution der Medien und der Medienkontrolle im postsowjetischen Russland
}

\author{
Von Anna Amelina
}

Zusammenfassung: Dass der politische Einfluss auf mediale Praktiken im postsowjetischen Russland heute teilweise dramatische Formen angenommen hat, hängt nicht zuletzt mit dem kontinuierlichen Wandel der Medienkontrolle zusammen. Die Frage nach den gesellschaftlichen und medialen Voraussetzungen einer solchen Medienentwicklung muss umso dringender untersucht werden. Am Beispiel der Evolution der Fernsehstrukturen im (post-)sowjetischen Russland zwischen 1970 und 2006 wird nun gezeigt, in welcher Weise die für die Sowjetunion typische Form der Propagandakommunikation ab 1985/86 durch neue Formen der Medienkommunikation ersetzt wird. Mit Hilfe empirischer Daten wird beschrieben, wie neben den Medienstrukturen auch die Formen der Medienkontrolle sich ändern. Die Darstellung wird in eine makrosoziologische Perspektive eingebettet, die den Zusammenhang von Medienevolution und gesellschaftlicher Strukturierung in der ehemaligen Sowjetunion betont.

\section{I.}

Jede zweite Stunde strahlen TV-Sender in Russland ${ }^{1}$ Nachrichten aus. Jede zweite Stunde werden die Zuschauer über die Aktivitäten des Präsidenten der Russischen Föderation informiert (ДЁик/НазароЁа 2002, S. 89) ${ }^{2}$. Bereits ab dem Jahr 2001, nach dem Wechsel der Medieneigentümer des mächtigen privaten Fernsehsenders NTV und der Auflösung des anderen bedeutenden privaten Fernsehsenders, TV-6, fällt die Berichterstattung über die Arbeit des Präsidenten und der Regierung sowohl in den staatlichen (Pervij Kanal ${ }^{3}$, Rossija, TVZ) als auch in den privaten TV-Sendern mit überregionaler Reichweite (NTV, TNT, STS, RenTV) positiv aus. Die Einflussnahme auf die redaktionelle Linie und die journalistische Selbstzensur sind die wesentlichen Mechanismen, die die Entscheidungen der Fernsehredaktionen im Themenbereich Regierungspolitik heute beeinflussen. Allerdings kann nicht behauptet werden, dass die Medienkontrolle (im Sinne der Einflussnahme auf redaktionelle Arbeit) erst von der Regierung der Russischen Föderation unter Putin als legitimes Politikinstrument angesehen wird. Dass die Medienlenkung ein notwendiges Instrument politischer Machtausübung ist, wurde bereits von Vladimir Lenin im Artikel »Über die Pressefreiheit « ([1905]1958) formuliert. Nach der Machtergreifung der Arbeiter- und Bauernpartei der Bolschewiken im Jahre 1917 und der Gründung der Sowjetunion wurde diese Idee leitend für die sowjetische Medienpolitik. Sie blieb prägend für die Logik der medialen Entwicklung sowohl in der Sowjetunion als auch im postsowjetischen Russland. Die Frage, die in diesem Aufsatz zu beantworten sein wird, lautet deshalb: Wie haben sich die medialen

1) Mit Russland ist hier und im Folgenden der Staat Russische Föderation gemeint. Beide Bezeichnungen gelten nach dem Artikel 1 der Verfassung der Russischen Föderation als Synonym. Bis Ende 1991 war die Russische Föderation eine Republik der Sowjetunion, die insgesamt aus 16 Republiken bestand.

2) Seit 2003 wird die Fernsehlandschaft in der Russischen Föderation von 9 führenden (sowohl staatlichen als auch privaten) TV-Sendern bestimmt, die aus Moskau in den Großteil der Regionen der RF senden (Pervij Kanal, Rossija, NTV, Sport, STS, Ren-TV, Kultura, TVZ, TNT). Außerdem existierten schon zu dieser Zeit über 1600 regionale, sowohl staatliche als auch private, Fernsehsender (ШарикоЁ 2003a; 2003b).

3) Zum jetzigen Zeitpunkt gehören dem Staat 51 Prozent der Aktien des Senders. 
Logiken und Strukturen im postsowjetischen Russland verändert? Wie kann der Wandel der Medienstrukturen - hier: am Beispiel des Fernsehens - analysiert werden?

Es existieren nur wenige theoretische Zugänge, die für die Beschreibung und die Erklärung der medialen Veränderungsprozesse in den osteuropäischen Ländern hilfreich sind. Die ersten theoretischen Ansätze der frühen 90er Jahre des 20. Jahrhunderts, die umfassende Transformationsprozesse in Osteuropa zu erklären suchten, gingen noch von der Zielgerichtetheit der Transformation aus. Sowohl die neoliberalen als auch die modernisierungstheoretischen Transformationskonzepte definierten Transformation als Einführung der demokratischen und marktwirtschaftlichen Institutionen nach westlichem Vorbild (Ettrich 1992; Voltmer 2000; Zapf 1994). Vor allem die Akteure, Implementierungsvorgänge und Geschwindigkeit der Transformation waren für diese Ansätze untersuchungswert. Während die neoliberalen Ansätze sich vor allem mit der wirtschaftlichen und politischen Transformation befassten, hatten sich modernisierungstheoretische Konzepte der frühen 90er Jahre des letzten Jahrhunderts u.a. auch den Untersuchungen des Medienwandels in postsozialistischen Ländern gewidmet (Androunas 1993; Murray 1994). Für diese empirischen Untersuchungen der Medientransformation war die Frage zentral, wie begünstigend der Medienwandel für die Ingangsetzung der marktwirtschaftlichen und demokratischen Institutionen ist. Danach hatten sich die Medien von den unerwünschten Formen der sozialen Kontrolle, wie politische oder wirtschaftliche Einflussnahme, zu trennen und die erwünschten Formen der sozialen Kontrolle, wie die Vorgaben der öffentlichen Meinung, zu institutionalisieren. Die reale Entwicklung postsozialistischer Medienlandschaften hat diese teleologischen Konzepte schnell in Frage gestellt. Vor allem die Idee der Zielgerichtetheit der Transformationsprozesse, die Vernachlässigung der Rolle der regionalen Strukturen sowie die universalistische, homogenisierende Perspektive der modernisierungstheoretischen Konzepte sind in diesem Zusammenhang kritisiert worden (Колцова 1999).

Wie kann nun der Medienwandel im postsowjetischen Russland so konzeptualisiert werden, dass die Schwierigkeiten der modernisierungstheoretischen Transformationsansätze überwunden werden? Eine solche Konzeptualisierung erfordert die Berücksichtigung von zwei theoretischen Zugängen. Zum einen ist hier eine mediensoziologische Perspektive erforderlich, die die wichtige Frage nach den Beziehungen zwischen den Medien und den politischen Instanzen klären kann. Zum anderen sollen die nicht-teleologischen makrosoziologischen Annahmen über den gesamtgesellschaftlichen Wandel im postsowjetischen Russland formuliert werden, weil dieser (neben globalen Entwicklungen) den Rahmen für den Medienwandel vorgibt.

\section{II.}

Für die Formulierung der wichtigsten mediensoziologischen These dieses Aufsatzes war zunächst die einfache Beobachtung leitend, dass die Formen der Medienkommunikation in der Sowjetunion sich von Medienlandschaften in den westlichen Ländern unterschieden haben (Thomaß/Tzankoff 2001). Es drängte sich die Vermutung auf, dass die Medienkommunikation in den sozialistischen Ländern, vor allem in der Sowjetunion, als Propaganda verstanden werden kann. Wie kann also der Begriff der Propaganda bestimmt werden? Diese Frage lässt sich durch den Rückgriff auf die bedeutenden Propagandatheoretiker durchaus erfolgreich beantworten. So arbeitete der bekannteste Theoretiker der Propaganda Harold Lasswell (1927; 1995) mit den Annahmen, die dem psychologischen Stimulus-Response-Modell der Kommunikationsforschung sehr nahe standen. Die konstruktivistische Reformulierung dieser Annahmen von Klaus Merten (2000) erlaubt uns Propaganda als Kommunikationstyp zu konzeptualisieren, der seine Operationsweise entlang einer spezifischen Entscheidungsregel ausrichtet. Nach dieser Regel kann das Publikum alle Medienstimuli unverändert aufnehmen 
und als Handlungsanweisungen übernehmen. So definiert Merten Propaganda als »eine Technik der Akzeptanz der angesonnener Verhaltensprämissen, bei der die kommunizierte Botschaft durch Reflexivisierung generalisierte Wahrheitsansprüche erzeugt, deren Akzeptanz durch Kommunikation latenter Drohpotentiale sichergestellt wird.« (Merten 2000, S. 161ff.)

Ein zentrales Merkmal der Propagandakommunikation ist organisatorische Unterordnung der Propagandamedien unter den Auftraggebern der Propagandabotschaften. Als Produktionsinstanzen von Propaganda werden hier die Redaktionen der Verbreitungsmedien (Presse, Fernsehen und Radio, aber auch Nachrichtenagenturen und Verlage) verstanden. Weil diese von einem Propagandaauftraggeber (wie z.B. noch vor 25 Jahren von der KPdSU in der Sowjetunion) geleitet werden, können seine Entscheidungskriterien Eingang in die redaktionelle Routinen der Propagandamedien finden. So kann die inhaltliche Ausrichtung der redaktionellen Arbeit durch den Propagandaauftraggeber vorgegeben werden. Dies impliziert auch, dass die zentrale journalistische Trennung zwischen dem Fakt und dem Kommentar auf der Ebene der Darstellungsroutinen in Propagandamedien nicht vorhanden ist.

Die gesellschaftliche Funktion von Propagandakommunikation besteht in erster Linie in der Reproduktion der Macht des Propagandaauftraggebers. So hatten in der Sowjetunion die Propagandamedien eine einzige, für alle sozialen und psychischen Einheiten verbindliche Realität zu artikulieren und die gesellschaftliche Position der KPdSU zu legitimieren. Die Auflösung des sowjetischen Gesellschaftskontexts wurde erst dann möglich, als Propaganda Ende der 80er Jahre des letzten Jahrhunderts mit einer neuartigen Form der Medienkommunikation zu konkurrieren begann. Erst, als der herkömmliche Aufbau der sozialen Ordnung durch neue aktualitätsbezogene Medien kritisiert wurde, konnte der intensive Wandel der gesellschaftlichen Strukturen in der UdSSR einsetzen.

Die neuen Medienstrukturen, die sich im postsowjetischen Russland entwickelt haben, ähneln einem Typ der Medienkommunikation, der als modernes System der Massenmedien bezeichnet wird (Luhmann 1996; Marcinkowski 1993; Weischenberg 1994). Eine solche systemtheoretisch-konstruktivistische Konzeptualisierung der modernen Medien bringt Einsichten in die innere Logik und Strukturen der Medienkommunikation. Aus dieser Perspektive werden moderne Medien als ein gesellschaftliches Teilsystem bzw. Funktionssystem definiert, dessen Operationsweise sich an die Prinzipien der Neuheit und der Aktualität ausrichtet $^{4}$ (Luhmann 1996). Die Medieninhalte werden in der Redaktion der Presse, des Radios und des Fernsehens sowie in Nachrichtenagenturen und Verlagen produziert. In diesen organisatorischen Instanzen wird die Selektion der Medieninhalte vorgenommen. Kennzeichnend ist, dass diese Selektionsprozesse nach den spezifisch massenmedialen Entscheidungskriterien kanalisiert werden (Blöbaum 1994, S. 277). Zu diesen zählen beispielsweise 1) Routinen der journalistischen Informationssuche (Techniken der Interviewführung, der Archivarbeit, des investigativen Journalismus u.a.), 2) Routinen der Informationsüberprüfung (Techniken der Arbeit mit Daten, mit Informanten, Techniken der Archivarbeit), 3) Routinen der Informationsselektion (zu ihnen zählen solche Selektionskriterien wie Aktualität, Neuheit, Konflikte, Skandale, Personen, Quantitäten, regionale Nähe usw.) und 4) Routinen der Informationsdarstellung (z.B. unterschiedliche Darstellungsformate im Fernsehen). Wichtig ist, dass massenmediale Thematisierungsleistung sich durch grundsätzliche Offenheit auszeichnet. So kann prinzipiell alles, was von massenmedialen Produktionsinstanzen als >informativ< eingestuft wird, in die Berichterstattung der Medien aufgenommen werden.

4) Niklas Luhmann spricht in diesem Zusammenhang von der Ausdifferenzierung einer binären Opposition, die er als Differenz zwischen Information und Nicht-Information bestimmt (Luhmann 1996, S. 42ff.). 
Die Logik des Mediensystems lässt sich genauer beschreiben, wenn wir das Publikumsbild unter die Lupe nehmen, das in den massenmedialen Organisationen gepflegt wird. Im Unterschied zu den Propagandamedien, die das Publikum als »willenlose Masse« definieren, wird hier von der Existenz des differenzierten Publikums ausgegangen. Für die Erzeugung ihrer Publikumsbilder verfügen die Medienorganisationen über mehr oder weniger ausgefeilte Instrumente, wie Leserbefragungen in der Presse und Einschaltquotenmessung im Fernsehen. Diese liefern die Konstruktionen der Publikumswünsche, die dann in den redaktionellen Entscheidungsprozessen berücksichtigt werden (Marcinkowski 1993, S. 83ff.).

Aus der aktualitätsbezogenen Logik der Massenmedien lässt sich ihre gesellschaftliche Funktion ableiten. So geht Luhmann (1996) davon aus, dass Massenmedien für die Gesellschaft eine Annahme bereitstellen, dass eine gemeinsame Hintergrundrealität erzeugt wird, auf die sich alle sozialen und psychischen Einheiten beziehen können. Allerdings sind diese Realitätsausschnitte und gesellschaftlichen Beschreibungen, die Massenmedien produzieren, nicht konsenspflichtig und können von Rezipienten immer unterschiedlich interpretiert werden. Im Unterschied zur Propaganda werden von einem Mediensystem also nicht eine singuläre gesellschaftliche Realität, sondern multiple gesellschaftliche Wirklichkeiten erzeugt.

Mit Hilfe des hier geschilderten Zugangs lässt sich der Medienwandel im postsowjetischen Russland als der Wechsel oder als Konkurrenz von zwei Typen der Medienkommunikation - Propaganda und Mediensystem - konzeptualisieren. Dabei soll berücksichtigt werden, dass erster Kommunikationstyp mit den politischen bzw. parteilichen Instanzen, die als Propaganda-Auftraggeber fungieren, extrem eng auf der Ebene der Entscheidungsprozesse verknüpft ist. Der zweite Kommunikationstyp - das Mediensystem - steuert eigene Selektionsprozesse sowohl auf der organisatorischen als auch auf der thematischen Ebene relativ autonom. Auf der Basis der empirischen Beobachtungen lässt sich in diesem Zusammenhang die Hypothese formulieren, dass die Propagandakommunikation, nach dem Beginn der umfassenden gesellschaftlichen Reformen in der Sowjetunion, sich im Auflösungsprozess befindet. Mit der Entstehung der Strukturen eines Mediensystems können Konkurrenzprozesse zwischen beiden Medienformen beobachtet werden.

\section{III.}

Das Interesse an konkreten sozialen Vorgängen des Medienwandels erfordert auch eine makrosoziologische Perspektive. Die makrosoziologische Konzeptualisierung des gesamtgesellschaftlichen Wandels als zielgerichtet oder als offen, als universalistisch oder als partikularistisch, als pfadabhängig oder als nicht-pfadabhängig prägt die Beschreibung des Medienwandels, der hier als ein Teilprozess der gesamtgesellschaftlichen Transformation eines postsowjetischen Gesellschaftsraums verstanden wird.

Der Rückgriff auf die Differenzierungstheorie (Luhmann 1998) und auf die Theorie der multiplen Moderne (Eisenstadt 1992; 2000) erlaubt hier, den gesamtgesellschaftlichen Veränderungsprozess im postsowjetischen Russland zu bestimmen. So bietet der Begriff der Differenzierungsform die Möglichkeit, das strukturelle >Erbe< der postsozialistischen Länder zu berücksichtigen. Die Transformationsprozesse werden nach dem differenzierungstheoretischen Ansatz als Aus- und Entdifferenzierungsvorgänge erfassbar, die reflexiv und ohne vorgegebenes Ziel ablaufen. Jedoch kann hier Luhmanns Annahme von der weltweiten Durchsetzung einer bestimmten Differenzierungsform - der funktionalen Differenzierung (Luhmann 1975; Stichweh 2000) - nicht ohne weiteres übernommen werden, weil sie universalistische und homogenisierende Aussagen der Modernisierungstheorie implizit mitführt. Plausibler als Idee der weltweiten funktionalen Differenzierung scheint die Annahme der regional unterschiedlich ablaufenden Differenzierungsprozesse zu sein, die aus den The- 
sen über die multiple Moderne von Shmuel N. Eisenstadt ableitbar ist ${ }^{5}$. So wären aus seiner Perspektive die postsozialistischen Transformationsprozesse als regional spezifisch verlaufende, nicht-universalistische Vorgänge einzustufen. In Anschluss an dieses Konzept schlage ich vor, von dem Wechsel der Dominanzverhältnisse zwischen zwei Differenzierungsformen im postsowjetischen Raum zu sprechen: Während eine Differenzierungsform - die so genannte Organisationsgesellschaft - der Erosion unterliegt, aber nicht verschwindet, bilden sich hier die Strukturen einer anderen Differenzierungsform aus - der Differenzierung in die Funktionsbereiche. Dieser offene, aber zugleich pfadabhängige Wandel der Dominanzverhältnisse konditioniert die Formen, die sich im Laufe des Medienwandels im postsowjetischen Russland herauskristallisieren.

Dieser Zugang hebt zudem hervor, dass gesellschaftliche »Probleme «, die sich in Bezug auf gesellschaftliche Funktionsbereiche herausbilden, nicht als universelle und zugleich weltweit gültige gedacht werden können. In Anschluss an Eisenstadt (1990, S. 255) lässt sich formulieren, dass gesellschaftliche Probleme und Problemlösungen regional, d.h. zivilisatorisch, variabel sind. So hängt das »Bezugsproblem der sozialen Komplexität« der massenmedialen Ausdifferenzierung im neuen Russland mit dem Umbau der sozialen Ordnung in der Sowjetunion / im neuem Russland zusammen. Beide gesellschaftlichen Differenzierungsformen sollen nun kurz vorgesellt werden.

Die gesellschaftlichen Strukturen der Sowjetunion können treffend mit der These der Organisationsgesellschaft beschrieben werden, die von Detlev Pollack (1994) für die Analyse gesellschaftlicher Strukturen der DDR eingeführt wurde. Ähnlich wie in der DDR wurde auch in der Sowjetunion ein Versuch unternommen, die Gesamtgesellschaft als Organisation einzurichten. Weil die kommunistische Partei ihre eigene Struktur, ihr Personal und ihre Entscheidungsregel auf die Gesamtgesellschaft übertrug, wurde für sie die Logik einer einzelnen Organisation verbindlich. Insbesondere der Staat war dem Parteiapparat untergeordnet. Die Mitglieder des Parteiapparats waren zugleich Inhaber der wichtigsten Staatsposten: des Staatsoberhaupts, der Minister usw. Zugleich befand sich der Parteiapparat außerhalb des Staates - über ihm (Sinowjew 1981, S. 245).

Primär differenzierte sich die sowjetische Gesellschaft in Zentrum - die Partei (insbesondere die obersten Gremien der KPdSU ${ }^{6}$ - und in die Peripherie, die Parteiumwelt. Weil im Zentrum wichtige Einflussmittel, wie Charisma, Prestige, physische Gewalt- und materielle Belohnungsmittel, konzentriert waren, konnten die Vorgaben und Anweisungen des Parteiapparats an jeden beliebigen Punkt der Gesellschaft übertragen werden ${ }^{7}$. Auf diese Weise

5) Eisenstadt geht von den weltweit unterschiedlichen Zivilisationspfaden aus, die sich in der s.g. Achsenzeit (etwa 800 bis 200 v. Christus) herausgebildet haben. Die Pfade wurden nach ihm durch jeweils unterschiedliche zivilisatorische Traditionen festgelegt, wobei er unter Tradition nichts anderes als ein spezifisches kulturelles Programm oder spezifische kulturelle Kodierung versteht (Eisenstadt [1980]1982, S. 36). Die Annahme der Möglichkeit unterschiedlicher zivilisatorischer Wege kann aus differenzierungstheoretischer Perspektive als These der regional unterschiedlichen Differenzierungsformen interpretiert werden.

6) Zum Parteiapparat zählten die Parteifunktionäre des Rayons-, Stadt-, Gebiets- und Republikkomitees und, auf der Unionsebene, das Zentralkomitee der Partei (ZK der KPdSU). Der Parteiapparat war hierarchisch aufgebaut. An seiner Spitze befand sich der Generalsekretär des ZK der KPdSU. Er leitete das Zentralkomitee der KPdSU, aus dessen Mitgliedern das so genannte Politbüro rekrutiert wurde. Die unterste Ebene war von Verantwortlichen Mitarbeitern in Regionen und Industrie besetzt. Zu betonen ist, dass der Parteiapparat sich relativ autonom von anderen Parteistrukturen reproduzierte.

7) Durch Kontrolle der Staatsfunktion und des polizeilich-repressiven Apparats besaß der Parteiapparat die gesellschaftsweite Befehlsmacht. Er konnte die Fortbewegung aller Individuen (Inlandpass) kontrollieren. Zugleich wurden die Staatsgrenzen strengstens überwacht. Der Parteiapparat besaß zudem das Monopol auf die Produktion der Wahrheiten und das Monopol auf die Produktion der materiellen Güter. 
konnten verschiedene Adressaten zur Ausführung gleicher Selektionsleistungen gesellschaftsweit motiviert werden. Diese Konzentration generalisierter Einflussmittel im Zentrum begünstigte eine schnelle Verbreitung von Homogenisierungstendenzen, die den Zustand geringer gesellschaftlicher Komplexität herbeiführten (Sinowjew 1981: S. 307). Weil aber gesellschaftliche Komplexität durch eine einzige Organisation in der Gesellschaft nicht immer reduziert werden konnte, entstand in der Gesellschaft ein Bruch zwischen formellen und informellen Strukturen. Zum einen kompensierten die informellen Strukturen, wie persönliche Netzwerke, Steuerungsdefizite der Organisationsgesellschaft. Zum anderen aber boten sich verlässliche Mechanismen des Zugangs zu den materiellen und symbolischen Ressourcen (Êîsäîînêèé 2000).

Während die Strukturen der sowjetischen Organisationsgesellschaft seit Ende der 80er Jahre des letzten Jahrhunderts mehr und mehr der Erosion unterliegen (sich jedoch nicht unbedingt endgültig auflösen), kann zunehmend die Herausbildung funktionaler Logiken beobachtet werden. Bekanntlich bezeichnet der Begriff der funktionalen Differenzierung den Sachverhalt, dass sich innerhalb einer Gesellschaft Teilbereiche herausbilden, die für einen Problemabschnitt in der Gesellschaft eine spezifische Funktion erfüllen (Luhmann 1998). Jeder dieser Teilbereiche (Politik, Recht, Wirtschaft, Wissenschaft, Kunst, Massenmedien, Familie, Krankenbehandlung, Sport, Religion usw.) konstituiert sich in Orientierung an ein jeweils besonderes Bezugsproblem, wobei die Bereiche einander nicht untergeordnet sind ${ }^{8}$.

Solche Ausdifferenzierungsprozesse wurden im postsowjetischen Gesellschaftsraum erst ab dem Jahre 1991 möglich, als das Monopol der KPdSU und des KGBs verschwand und der Zerfall der Sowjetunion das Ende politischer Repressalien besiegelte. Das politische Feld der Ex-Sowjetunion wurde durch die Gründung mehrerer neuer Staaten durchkreuzt. Auch im Staat der Russischen Föderation, der sich als Nachfolger der Sowjetunion erklärte, fanden vielfältige politische Ausdifferenzierungsprozesse statt. So konnte mit der Einführung der Mehrparteienregime die Trennung zwischen Regierung und Opposition institutionalisiert werden. Es wurden Wahlverfahren eingeführt, die sich durch die reale Konkurrenz an politischen Programmen auszeichneten. Neben neuen politischen Parteien gründeten sich zahlreiche Stiftungen und Nichtregierungsorganisationen. Nach dem Zusammenbruch des sowjetischen Regimes nahmen auch die formalen demokratischen Rechte der Bürger zu, obgleich die unterschiedlichsten Interessengruppen in den Kampf um die praktische Durchsetzung dieser Rechte verwickelt waren. Mit den politischen Ausdifferenzierungen waren rechtliche Ausdifferenzierungen verknüpft. So wurden im Laufe der letzten 20 Jahre neben der Verfassung eine Vielzahl neuer Gesetze und Codices verabschiedet. Angemerkt sei, das $30 \%$ der gesetzlichen Regelungen einander widersprechen. Gleichzeitige Gültigkeit mehrerer Rechtsnormen erlaubt den zuständigen Richtern im Kontext der kontinuierlichen Korruption vorteilhaftere Regelungen in Verfahren zu verwenden.

Neben den politischen und rechtlichen Ausdifferenzierungsprozessen wurden auch wirtschaftliche Ausdifferenzierungsprozesse intensiviert. Die Einführung des Privateigentums ermöglichte die Herausbildung solcher wirtschaftlichen Organisationen wie Unternehmen. Der stärkere Anschluss an das globale Wirtschaftsfeld ermöglichte das Agieren der ausländischen Unternehmen auf den russischen Märkten. Die gleichzeitige Privatisierung des sowjetischen Staateigentums veränderte die wirtschaftliche Landschaft des neuen Russlands enorm: Auf der einen Seite wurden viele Zweige der Großindustrie, wie die chemische Industrie und der Maschinenbau zerstört, auf der anderen Seite wurde die Rohstoffindustrie ge-

8) Im Anschluss an Eisenstadt muss jedoch hervorgehoben werden, dass gesellschaftliche Probleme, die mit Herausbildung der Funktionsbereiche zusammenhängen, höchst wahrscheinlich regional spezifisch ausfallen. 
winnbringend reorganisiert. So ist russische Markt eher durch eine Dominanz der Monopole zu charakterisieren, während das kleine und mittlere Unternehmertum sich langsamer entwickeln.

Die Hinweise auf vielfältige Differenzierungsprozesse im postsowjetischen Raum zeugen jedoch nicht notwendigerweise von Angleichungsprozessen zwischen der Russischen Föderation und ihren europäischen und amerikanischen Vorbildern. Eher ist hier eine komplexe Dynamik der Adaption und Ablehnung verschiedener institutioneller Muster beobachtbar. So lässt sich beispielsweise im politischen System eine formale Einführung der demokratischen Institutionen beobachten, doch diese Prozesse werden häufig von einer Imitation der Reformen begleitet (Ëåâààà 2003). Insbesondere staatliche Instanzen neigen zur Verwendung demokratischer Semantik, wobei formal implementierte demokratische Strukturen real nicht gefördert werden.

Meine Annahme ist, dass die Übergangsphase zwischen Organisationsgesellschaft und funktionaler Differenzierung als nicht-theleologischer Prozess einzustufen ist. Eher bringt sie einen neuartigen Kontext hervor, in dem die charakteristischen Merkmale beider gesellschaftlicher Formen zum Ausdruck kommen: Zentralisierung, Geschlossenheit und Trennung zwischen formellen und informellen Strukturen sind demnach kennzeichnend für eine Organisationsgesellschaft; funktionale Differenzierung, Offenheit und Minimierung der Distanz zwischen formellen und informellen Strukturen - für die funktionale Differenzierungsform. Diese spezifischen Eigenschaften beider Gesellschaftsformen sind als Antinomien zu interpretieren, die während der Übergangsphase Prozesse konditionieren, die sich in gesellschaftlichen Teilbereichen abspielen.

Ein zentrales Argument meiner Konzeptualisierung des Medienwandels ist die These, dass sich die jeweilige Differenzierungsform durch einen spezifischen Typ der Medienkommunikation auszeichnet: Während für die sowjetische Organisationsgesellschaft Propaganda einen zentralen Modus der medialen Kommunikation darstellt, konstituiert sich in einer funktional differenzierten Gesellschaft ein Mediensystem, das sich relativ autonom von anderen gesellschaftlichen Teilsystemen auf der Grundlage seiner spezifischen Eigenlogik reproduziert. Folglich könnte der Medienwandel im postsowjetischen Russland als Erosion (d.h. Entdifferenzierung) von Propaganda und Ausdifferenzierung eines Funktionssystems der Massenmedien verstanden werden. Wenn jedoch berücksichtigt wird, dass die Verlagerung der Dominanzverhältnisse zwischen beiden gesellschaftlichen Differenzierungsformen eine kontinuierliche und langfristige Transformation aller gesellschaftlicher Teilbereiche voraussetzt, die durch Überraschungen, Konflikte und Enttäuschungen charakterisierbar ist, ist der rasch ablaufende postsowjetische Medienwandel der letzten zwei Jahrzehnte nicht als Wechsel, sondern als Konkurrenz zwischen Propaganda und autonomen Medien zu verstehen. Dieser Wandel soll hier nun auf der Grundlage des empirischen Materials beschrieben werden.

\section{IV.}

Meine empirische Untersuchung des medialen Wandels im postsowjetischen Russland von 1970 bis 2006 - hier am Beispiel des Fernsehens - erlaubt fünf Zeiträume herauszugliedern, die durch spezifische Konfigurationen der Medienstrukturen gekennzeichnet sind. Zunächst soll hier der Status quo des sowjetischen Fernsehens (1970-1985) nachgezeichnet werden ${ }^{9}$.

9) Neben der Analyse der Sekundärquellen wurden im Jahre 200132 Interviews mit den Vertretern des russischen Fernsehens durchgeführt. 


\section{Unterordnung des Fernsehens unter den Parteiapparat (1970-1985)}

In den 70er Jahren des letzten Jahrhunderts war das sowjetische Fernsehen nach den staatlichen Verwaltungsebenen strukturiert ${ }^{10}$ : Auf der Unionsebene existierte das Zentralfernsehen, auf der republikanischen Ebene - Fernsehen der Republiken, auf der städtischen Ebene - s.g. Örtliche Studios. Das Gosteleradio - Staatskomitee für Fernsehen und Hörfunk - kontrollierte den Fernsehbetrieb auf diesen Ebenen auf dem Territorium der gesamten Sowjetunion. Aufgrund seiner fast landesweiten Reichweite nahm das Zentralfernsehen eine herausragende Rolle für die Produktion der Realitätsannahmen über das sowjetische Leben an ${ }^{11}$.

Obwohl das Fernsehen formal durch staatliche Behörden geleitet wurde, wurde seine Steuerung real durch den Parteiapparat übernommen. Das Sekretariat des Zentralkomitees der KPdSU war der einflussreichste Ausschuss der Partei, der sich der Kontrolle von Gosteleradio widmete. Insbesondere zwei Abteilungen - Agitprop (Abteilung für Agitation und Propaganda) und Abteilung für Parteiarbeit und Ideologie - nahmen die Überwachung von Gosteleradio wahr. Eine andere mächtige Behörde, das Zensurorgan arbeitete in Fragen der Fernsehkontrolle mit dem ZK-Sekretariat zusammen. Die wichtigsten Entscheidungen über die Fernseharbeit wurden im Agitprop getroffen. Hier wurden Finanzierungs- und Personalfragen geklärt, Richtlinien für die Produktion von Inhalten verabschiedet und anschließend an Gosteleradio weitergeleitet.

Die mehrfache Mitgliedschaft der Fernsehleitung bildete eine weitere Schnittstelle zwischen der parteilicher und staatlicher Organisationsstruktur: Zu seinem Amt wurde der Leiter des Gosteleradio von einer Staatsbehörde, dem Obersten Sowjet der UdSSR, ernannt. Als Kabinettsmitglied und Staatssekretär war er dem Ministerrat, also einem staatlichen Gremium, unterstellt. Zugleich hatte er die Mitgliedschaft des Zentralkomitees der KPdSU inne. Ähnliche Mitgliedschaftsverteilung galt auch für das Leitungsorgan des Gosteleradio - fürs Kollegium. Formell war das Kollegium einer staatlichen Instanz - dem Ministerrat der UdSSR - unterstellt. Die Mitglieder des Kollegiums waren jedoch zugleich Mitglieder des Zentralkomitees der KPdSU (Müller 2001, S. 39).

Die Kopplung der Fernsehorganisation an den Parteiapparat erlaubte den Transfer der parteilichen Kriterien in die Redaktionen des Zentralfernsehens. Danach mussten sowjetische Fernsehjournalisten und -redakteure ihre Arbeitsroutinen entlang der Kriterien, wie Parteilichkeit, Objektivität/Sachlichkeit, Volksnähe und Glasnost/Offenheit ausrichten.

Diese Prinzipien hat Wladimir Lenin in Anschluss an Wilhelm Liebknecht in seiner Pressetheorie ([1905]1958) formuliert. In diesem Konzept stellte Parteilichkeit ein Prinzip dar, nachdem jede journalistische Veröffentlichung als >offenkundige Vervielfältigung der Klassen-Weltanschauung< verstanden werden musste. Danach sollte die Presse offen für die Interessenverteidigung der Arbeiterklasse kämpfen. Das Prinzip der Objektivität/Sachlichkeit der Presse wich von dem westlichen Verständnis der journalistischen Objektivität ab. Die Definition der >sozialistischen Objektivität' beruhte auf dem Vertrauen in die Wissenschaftlich-

10) Mit der Entwicklung der technischen Infrastruktur des Fernsehens wurde in der Sowjetunion bereits in den 50er Jahren des letzten Jahrhunderts begonnen. Allerdings konnte die unionsweite Ausstrahlung erst in den 70er Jahren in Gang gesetzt werden, weshalb die Beschreibung des sowjetischen Fernsehens in diesem Artikel ab dem Jahr 1970 beginnt.

11) Das Zentralfernsehen war intern in 11 Hauptredaktionen gegliedert, die in den 70ern Jahren 4 und später 5 Fernsehprogramme produzierten. Dabei erreichten das I. und II. Fernsehprogramm zwischen 95 und 98\% der Bevölkerung der Sowjetunion. Das III. Programm konnte die Bevölkerung Moskaus und das Moskauer Einzugsgebiets empfangen. Das IV. Programm wurde auf dem europäischen Teil der Sowjetunion ausgestrahlt. Das V. Programm konnte man in Leningrad und im Leningrader Gebiet empfangen. 
keit der marxistisch-leninistischen >Theorie <. Nach dem Prinzip der Volksnähe sollte Presse einen Massencharakter aufweisen. Die >Verbundenheit zwischen der Presse und den Massen< sollte sich in den steigenden Auflagezahlen der Zeitungen widerspiegeln. Das letzte der Lenin'schen Kriterien war das Glasnost-Prinzip bzw. das Prinzip der Offenheit. Dieses Kriterium beinhaltete die Forderung nach der breiten Propaganda der positiven Errungenschaften des sowjetischen Lebens (Прайс 2000, S. 102). Später, am Ende der Sowjet-Ära fungierte das Glasnost-Prinzip als Hauptbegründung für die Ingangsetzung der umfassenden gesellschaftlichen Reformen.

Damit die Arbeit der sowjetischen Journalisten - die Produktion der Propagandabotschaften - nicht von diesen Kriterien abwich, wurde ein mächtiger Zensurmechanismus institutionalisiert, der die Fernseharbeit steuerte ${ }^{12}$. So war jeder Fernsehredaktion mindestens ein Zensor zugeteilt worden, der unmittelbar vor der Ausstrahlung Durchsicht aller Inhalte vornahm. Insgesamt differenzierte sich die Zensur in a) ideologische und b) faktologische. Unter ideologischer Zensur wurde die Abstimmung der unveröffentlichten Fernsehinhalte mit den parteilichen Vorgaben verstanden. »Faktologische« Zensur beinhaltete Veröffentlichungsverbot historischer Fakten, die Konsistenz ideologischer Vorgaben gefährden könnten. Beispielsweise wussten sowjetische Rezipienten nichts über den Einmarsch sowjetischer Panzer in Prag im Jahre 1968. Selbst zu Beginn der Reformen, als im April 1985 der Tschernobyl Atom-Reaktor hochging, wurde darüber weder im Fernsehen noch in anderen Medien berichtet. Erst Monate später, nachdem tausende Bewohner bereits verstrahlt waren, hob der damalige Generalsekretär der KPdSU, Michail Gorbatschow, das Informationsverbot über die Tschernobyl-Katastrophe auf ${ }^{13}$. Der Zensurmechanismus verhinderte die Herausbildung des Bezugs zur Aktualität im Fernsehen und in den anderen Medien. Die Tatsache, dass im Fernsehen nur eine einzige Sendung (Abendnachrichten Vremja) live ausgestrahlt wurde, kann als Indikator dafür gewertet werden.

Die oben vorgestellten Mechanismen der Fernsehkontrolle (Monopolisierung, Zensur/Einwirkung au die redaktionelle Linie, Überwachung durch KGB und Parteiboten, Personaltransfer/Auswahl des Personals, Sanktionen) konnten erst nach Beginn von umfangreichen gesellschaftlichen Reformen in der noch bestehenden Sowjetunion geschwächt werden, als vom erneuerten Parteiapparat im Jahre 1985 die Politik von Perestroika und Glasnost initiiert wurde.

12) Das einwandfreie Funktionieren des Zensurmechanismus wurde durch so genannte Instrukteure gewährleistet. Sie waren Mitarbeiter einer speziellen Abteilung von Agitprop, der s.g. Sektion für Hörfunk und Fernsehen. Diese >Boten' waren für die Weiterleitung der Direktiven von Agitprop an alle Organisationsebenen des Fernsehens zuständig. Instrukteure hatten nicht nur journalistische Arbeit in den Fernsehredaktionen überwacht, sondern auch Redakteure und Journalisten ideologisch ausgebildet. Die umfassende Wirkungsweise dieses Selektionsmechanismus führte zur Herausbildung individueller Anpassungsstrategien, wie Selbstzensur und vorauseilenden Gehorsam.

13) Die Selektion der Realitätsausschnitte auf der Grundlage der parteilichen Richtlinien konnte jedoch nicht komplexitätsadäquat durchgeführt werden, weshalb in der Sowjetunion informelle (polizeilich verfolgte) Medien - so genanntes Samisdat - entstanden sind, die i.d.R. auf den Schreibmaschinen hergestellt wurden (vgl. Коломиец., 2001, S. 255f.). Jedoch konnten sich alternative Informationsquellen nicht im Bereich des Fernsehens herausbilden. Die Funktion der alternativen Medien bestand in der Herstellung einer zur offiziellen alternativen Wirklichkeit. Allerdings konnte diese nur auf der Wahrnehmungsebene existieren, weil öffentliche und private Kommunikation über Inhalte informeller Medien sofort sanktioniert wurde. 


\section{Thematische Öffnung des Fernsehens und Herausbildung des Prinzips der Aktualität (1986-1991)}

Der Beginn der Umgestaltungsphase, die, wie später offensichtlich wurde, den Niedergang des sowjetischen Imperiums einleitete, setzte mit dem berühmten Plenum des Zentralkomitees der KPdSU im April 1985 ein. Auf diesem Plenum stellt der Generalsekretär Michail Gorbatschow, sein Reformpaket vor, das insgesamt drei Säulen umfasste. Erstens sollte unter der Leitung der Partei und mit Hilfe der modernen Technologien Beschleunigung (Uskorenije) aller gesellschaftlichen Bereiche, vor allem der Wirtschaft, erreicht werden. Zweitens sollten alle Organisationsstrukturen der sowjetischen Gesellschaft umgestaltet werden, ein Vorhaben, das unter dem Begriff Perestroika bekannt wurde. Drittens sollten mit der Politik der Glasnost, die als Politik der gelenkten informationellen Öffnung verstanden wurde, Hindernisse auf dem Reformweg beseitigt werden. Zwar waren die Medieninstanzen durch die Politik der Glasnost von der Zusammenarbeit mit dem Zentralkomitee der KpdSU nicht entbunden, aber sie durften nun, mit dem Einverständnis der Parteiführung, neue Realitätsausschnitte, die nach der Ansicht der Parteiführung für die Durchsetzung der Reformen notwendig waren, thematisieren ${ }^{14}$.

Die Politik der Glasnost erlaubte es den Journalisten, Nachforschungen über die sowjetische Vergangenheit anzustellen. Auf der Grundlage von zuvor geheim gehaltenen Archivmaterialien wurden Themen wie Verbrechen der KPdSU sowie historische Fakten über Oktoberrevolution und über den Zweiten Weltkrieg in der Presse und im Fernsehen diskutiert. Offizielle Geschichtsentwürfe wurden intensivst hinterfragt. Nach dem Bekanntwerden der Verbrechen des kommunistischen Regimes war das Streben nach kommunistischer Zukunft nicht mehr ersichtlich. Zugleich ermöglichte diese Kritik die Entstehung alternativer Vergangenheits- und Zukunftsperspektiven. Auch die Entscheidungsvorgänge in der mittleren und höheren Ebenen der Parteihierarchie durften ab jetzt kritisch beleuchtet werden. Zunächst schien es, als würden die Medieninstanzen, wie gewohnt, die Anweisungen des Parteiapparats erfüllen - in diesem Fall umfassende gesellschaftliche Kritik zu üben. Doch schon nach wenigen Jahren wurde offensichtlich, dass nicht nur die Quantität der Kritik enorm zunahm, sondern auch, dass die Masse der kritischen Berichte den parteilichen Kriterien der Medienlenkung widersprach. Die thematische Öffnung des Fernsehens, die durch die Politik der Glasnost begünstigt wurde, erlaubte eine Infragestellung von allem Gewohnten, Vertrauten, Verbindlichen. Sie erlaubte eine besonders empfindsame Wahrnehmung gesellschaftlicher Kontingenz. Mit dem Anwachsen der kritischen Berichterstattung wurden plötzlich die Fragen laut: Warum existieren keine festen Vorgaben zur Bewertung des sowjetischen Lebens mehr? Sind mehrere Beschreibungen aktueller Ereignisse zugleich möglich? Können alte Lebensmuster ohne Sanktionen verändert werden? So übernahm das entstehende Mediensystem die Verarbeitung der spezifischen »Bezugsprobleme der gesellschaftlichen Komplexität« in der sich verändernden Organisationsgesellschaft: Es ermöglichte die Öffnung der Organisationsgesellschaft auf der Ebene der Kommunikationsthemen und die Pluralisierung der zeitlichen Horizonte im sowjetischen Gesellschaftskontext.

Durch die thematische Öffnung konnte ein Mehr an Information sowie verschiedenartige Informationen in die Bahn der sich herausbildenden Medieninstanzen gezogen werden. Als

14) Für die Parteiführung unter Gorbatschow stellte die Politik von Glasnost keinen selbstlosen Zweck dar. Seit Reformbeginn fanden innerhalb des Parteiapparats heftige Machtkämpfe statt. Die konservativen Mitglieder waren mit der Richtung der eingeschlagenen Reformen nicht einverstanden. Der Reforminitiator Gorbatschev gehörte dagegen dem liberalen Flügel innerhalb des Parteiapparats an. Gerade aufgrund der inneren Machtkämpfe im Parteiapparat kam den Medien im Reformprozess eine prominente Rolle zu. Sie sollten nach Vorgaben von Gorbatschow und seinen Anhängern die Notwendigkeit der Reformen in breiter Öffentlichkeit begründen (Засурский, И., 2001, S. 7f.) 
Indikator der Herausbildung einer genuin massenmedialen Eigenlogik kann sowohl die enorme Zunahme der Auflagezahlen der Presse als auch gravierende Zunahme der Live-Übertragungen im noch sowjetischen Fernsehen gelten: 1989 stieg die Tagesauflage von 8800 Zeitungen auf 280 Millionen Exemplare, die Tagesauflage der 1629 Zeitschriften stieg auf 220 Millionen; der Umfang der Live-Sendungen im Fernsehen nahm in den ersten zwei Jahren der Reformen (1986/1887) um ein 30-faches zu. Diese Zahlen plausibilisieren, dass Prinzipien der Neuheit und Aktualität für die neu entstehende massenmediale Kommunikation von zunehmender Wichtigkeit werden (Грабельников 2001, S. 94ff.; Муратов 2001, S. 30ff.).

Obwohl die Politik der Glasnost die informationelle Öffnung begünstigte, waren die Kontrollinstrumente des Fernsehens (und anderer Medien) in den ersten Jahren der Glasnost-Ära intakt, denn Berichterstattung sollte zwar offener werden, jedoch nach wie vor Parteivorgaben »umsetzen«. Die Trennung des Fernsehens von dem Parteiapparat auf der Organisationsebene war noch nicht vorhanden, was zur Ingangsetzung der Kontrollinstrumente in Fällen besonders abweichender Berichterstattung führte. So wurden die besonders kritisch eingestellten Fernsehjournalisten entlassen oder auf den Parteiversammlungen ermahnt, skandalträchtige Berichterstattung wurde zensiert, populäre Fernsehsendungen eingestellt ${ }^{15}$. Allerdings konnten diese Vorgänge durch die Presse kritisch enthüllt werden. Es erschien absurd, dass mit der Politik der Glasnost Kritik an vielen gesellschaftlichen Missständen geäußert werden durfte, aber nicht, wenn sie die obere Parteiführung betraf.

Diese Kritik führte zur zunehmenden Auflösung der parteilichen Kontrollmechanismen des Fernsehens. Die kritischen Fernsehjournalisten kündigten ihre Stellen in Redaktionen und gründeten eigene Presseeinrichtungen. Vor allem setzte diese Welle seit der Verabschiedung des Pressegesetzes 1990 ein. Die Kündigungen konnten nicht sanktioniert werden, aber gerade sie untergruben die Regeln der parteilichen Selektion des journalistischen Personals. Weil irgendwann viele Journalisten das Zentralfernsehen verlassen hatten, wurden die Personalressourcen dieser Organisation stark ausgedünnt.

Auch der Zensurmechanismus wurde zunehmend schwächer, weil die Ausübung der Zensur nach altem Muster ein zunehmendes Glaubwürdigkeitsproblem für die Parteiführung erzeugte. Sowohl ein Publikationsverbot als auch Journalisten-Entlassungen würden den Parteikurs selbst in Frage stellen. So wurde die umfassende Zensur zunehmend durch einzelne Telefonanweisungen von unterschiedlichen Teilorganisationen des Parteiapparats an die Fernsehleitung ersetzt. Zunehmend wurde auch innerhalb des Parteiapparats unklar, wer die Medienkontrolle herbeizuführen hat und welche Formen sie annehmen soll. So deutet gerade die Zunahme der Telefonanweisungen von unterschiedlichen Teilorganisationen des Parteiapparats an die Leitung des Zentralfernsehens auf die wachsende Dezentralisierungsprozesse innerhalb des Parteiapparats hin (Mickiewicz 1999, S. 115). Diese Dezentralisierung der Kontrolle erlaubte vielen Fernsehredakteuren schon Ende der 80er Jahre, sich den Anweisungen der Parteiführung zu widersetzen.

Auch der Aufgabenbereich der Zensurbehörde Glavlit wurde eingeschränkt. Schon 1987/ 1988 hat Glavlit auf ideologische und faktologische Zensur aller Informationsträger verzichtet. Seitdem befasste sich die Behörde mit der Überwachung der Staatsgeheimnisse, obwohl dieser Begriff nach wie vor sehr weit ausgedehnt werden konnte. Mit der Verabschiedung des revolutionären Pressegesetzes 1990 wurde die Zensur vollständig abgeschafft. Die Mitarbeit von Glavlit mit den Medieninstanzen erfolgte seitdem auf der Vertragsbasis. Nach dem Regierungsputsch 1991 wurde diese Organisation vollständig aufgelöst (Федотов 2002, S. 122).

15) Als Beispiel kann die Schließung der Nachrichtensendung TSN auf dem zweiten Programm gelten, das mehrfach zensiert und 1991 geschlossen wurde. 
So können wir rückblickend in diesen Jahren die Auflösung der Mechanismen des Personaltransfers, der Zensur, der Überwachung und der Sanktionen feststellen, die im Gesetz »Über Presse und andere Medien« ihre Kulmination fand. Zugleich konnte ab 1990 der Prozess der Verselbstständigung der journalistischen Organisationen einsetzen. Das Gesetz »Über Presse und andere Medien« von $1990^{16}$ hat nicht nur die Zensur aufgehoben, es erlaubte auch die Gründung von autonomen Medienorganisationen. Danach konnten die Redaktionen, die zuvor ihren Herausgebern, wie Gewerkschaften, Parteiinstanzen, Berufsverbänden usw. unterstellt waren, sich von ihnen trennen. Das Gesetz erlaubte erstmalig den Redaktionen, selbst als Herausgeber zu fungieren. Nun konnten Redaktionen als rechtliche Personen auftreten, die auf der Grundlage der Satzung tätig waren. Zudem führte das Gesetz die Registrierungsregeln für die Organisationen der Presse, des Radios und des Fernsehens ein. Neben Organisationen konnten ab jetzt auch private Personen die Herausgeberschaft übernehmen ${ }^{17}$ (Федотов 2002, S. 122f.).

Neben der Erosion der parteilichen Kontrollmechanismen des Fernsehens und Herausbildung des Prinzips der Aktualität beeinflusste auch die neue Art, wie Journalisten jetzt über ihre Arbeit reflektierten, die Herausbildung der genuin massenmedialen Eigenlogik der Informationsverarbeitung erheblich. Ende der 80er Jahre setzte sich im sowjetischen Journalismus die Idee der Medien als vierter Gewalt durch. Die Journalisten definierten sich nun als die Volksvertreter, die fähig sind, Entscheidungsprozesse politischer Machtinhaber zu beeinflussen. Diese Idee unterschied sich jedoch inhaltlich von der westlichen Semantik der Medien als public sphere. Im neuen Reformkontext »sahen (die sowjetischen Journalisten) ihre Aufgabe nicht in der Informierung des Publikums oder der Formierung eines glaubwürdigen Realitätsbildes, sondern in der Aufklärung, Agitation und Organisation der Massen zum Zwecke der wahren Werte und Ideale« (Засурский, И., 2001, S. 16). Nicht die Übermittlung von Fakten und Informationen, sondern Überwindung der Rückstände von kommunistischen Ideen in der Bevölkerung, wurde zur journalistischen Aufgabe. Die Übermittlung der neuen »richtigen« Ideen, wie Marktwirtschaft und Demokratie, wurde zur zentralen journalistischen Aufgabe in dieser Zeitperiode. Die neue Form der journalistischen Selbstbeschreibung erlaubte es, die zunehmende Autonomisierung der Massenmedien innerhalb des sich wandelnden sowjetischen Gesellschaftsraums zu legitimieren und zu begründen. Gleichzeitig wurde durch sie die Umbewertung der Rolle des Journalisten initiiert.

\section{Kommerzialisierung des Fernsehens und seine Trennung vom Staat auf der Organisati- onsebene (1991-1995)}

Im Prozess der Kommerzialisierung des Fernsehens entstand ein neuer Typ der Fernsehorganisation - der kommerzielle Fernsehsender. Während der erste kommerzielle TV-Sender »Fernsehkanal 2X2« (gegründet 1990 und privatisiert 1991) nur auf dem Territorium von Moskau und des Moskauer Gebiets sendete, erreichte der 1993 gegründete private Sender NTV schon den europäischen Teil der Russischen Föderation. Der andere 1993 gegründete private Fernsehsen-

16) Im Dezember 1991 wurde im neu gegründeten Staat Russische Föderation ein »Gesetz über die Massenmedien« verabschiedet, das zu Beginn von 1992 in Kraft gesetzt wurde. Er wurde von den gleichen Autoren, wie das Gesetz »Über die Presse und anderen Medien« von 1990 entworfen, war jedoch viel radikaler in der Durchsetzung des Rechts auf die Pressefreiheit, weil es nach dem Verbot der KPdSU entstanden ist.

17) Unmittelbar nach der Verabschiedung dieses Gesetzes herrschte in der sowjetischen Medienlandschaft eine unglaubliche Gründungseuphorie: Es wurde eine Vielzahl von Zeitungen und Zeitschriften gegründet. Weil die offizielle Registrierung erst nach der Verabschiedung des Pressegesetzes möglich wurde, gab es eine Welle der Herauslösung der Redaktionen von ihren Herausgeberinstanzen, wie dem Zentralkomitee der KPdSU, der Komsomolzenorganisation oder Gewerkschaften. Im März 1991 waren bereits 1800 Zeitungen und Zeitschriften registriert, von denen 870 Neugründungen waren (Грабельийков 2001, S. 98). Auch die informellen Alternativmedien konnten durch dieses Gesetz legalisiert werden. Im Fernsehen konnte sich dieser Trend erst seit 1991, nach dem Zerfall der Sowjetunion, im Zeitraum zwischen Ende 1991 und 1995, entwickeln. 
der TV-6 hatte zunächst nur einen kleinen Zuschauerkreis (500 tausend potentielle Zuschauer in Moskau), vergrößerte jedoch durch Einführung technischer Neuerungen den Publikumsumfang auf 67,7 Millionen Zuschauer in RF im Jahre 1996. Die neuen Fernsehsender wurden schlagartig weit über Moskau hinaus populär - nicht zuletzt, weil sie nach den neuen Formen der Informationsproduktion suchten. Sie ahmten ausländische Vorbilder nach, zum Beispiel NTV hat die Formen der Nachrichtenproduktion von CNN imitiert. Die neuen Sender kooperierten auch mit ausländischen Fernsehorganisationen: Telekanal 2X2 - mit dem englischen Super Channel; TV6 - mit dem amerikanischen Sender TNT. Zudem konnte TV-6 die Filmressourcen der elektronischen Bibliothek von CNN International nutzen. Neben den gesamtnational bedeutenden kommerziellen TV-Sendern, wie NTV und TV-6, entstand in dieser Phase eine hohe Anzahl regionaler kommerzieller Sender. 1991 wurde der erste Pay-TV-Sender gegründet. Im gleichen Jahr entstand die erste »Internationale Assoziation des Radios und Fernsehens « (MART), die insgesamt 70 regionale Fernsehsender vereinte. Im Jahr 1995 entstand ein anderer nicht-kommerzieller Fernsehverein, die »Nationale Assoziation der Fernsehsender« (NAT).

Die Kommerzialisierung des Fernsehens in Russischer Föderation zeichnete sich durch zwei wichtige Momente aus. Erstens wurde sie durch die Entstehung der (Fernseh-)Märkte, wie Markt der Fernsehwerbung, Markt der Unterhaltungsprogramme und der Nachrichtenmarkt, begleitet. Deshalb stieg der Anteil der Werbeinhalte in den Fernsehprogrammen der neuen Sender erheblich. Auch die Anteile der ausländischen (vor allem amerikanischen) Fernsehfilme haben sich vergrößert (Коломиец 2001, S. 180).

Zweitens führte die Kommerzialisierung des Fernsehens zur Schwächung der klassischen Kontrollmechanismen der Medien. Weil staatlicher Einfluss sich nicht auf die kommerziellen Fernsehorganisationen anwenden ließ, führte die Kommerzialisierung schließlich zur endgültigen Entkopplung des Fernsehens vom Staat auf der Organisationsebene. Denn der Kommerzialisierungsprozess erfasste auch die führenden staatlichen TV-Stationen Ostankino (ab 1994 umbenannt in ORT) und RTR. Die Entstehung der neuen kommerziellen Fernsehsender, die sich bereits nach ihrer Gründung an den marktrelevanten Kriterien orientierten, zwang auch die gesamtnationalen staatlichen Sender zur Konkurrenz um das Publikum. Denn jetzt befanden sich sie in einer neuen Situation, in der die staatliche Budgetfinanzierung kleiner und der Finanzierungsdruck zunehmend größer wurde. Die Konkurrenzsituation auf dem entstehenden Markt der Fernsehwerbung setzte beide staatlichen Sender (Ostankino und RTR) unter Druck. Sie waren gezwungen ihre Entscheidungskriterien zu überdenken.

Bemerkenswert ist, dass die Kommerzialisierung des staatlichen Fernsehens Ostankino mit der Legalisierung der Schattenstrukturen des Fernsehens einherging. Bereits ab 1991 wurden Werbeeinnahmen von Ostankino bezogen, jedoch in der Regel von den Redaktionsleitern privat eingenommen. Erst 1994 wurden diese Strukturen legalisiert. Der Sender wurde in eine Aktiengesellschaft ORT umgewandelt und zu 49 Prozent durch eine Reihe von Banken privatisiert, 51 Prozent der Aktien blieben dabei in staatlicher Hand ${ }^{18}$. Der andere Sender, RTR, blieb zwar nach wie vor im Besitz des Staats ${ }^{19}$, musste sich jedoch aufgrund der Budgetkürzungen größtenteils durch Werbeeinnahmen finanzieren ${ }^{20}$ (Zvik/Nasarova 2002, S. 47).

18) Nach der Umwandlung des Senders Ostankino in eine Aktiengesellschaft ORT konnte der Präsident nach wie vor über die Einstellung und Entlassung der Fernsehleitung entscheiden. Allerdings verzichtete der Staat, trotz des Besitzes vom Kontrollpaket der Aktien, vollständig auf die Finanzierung des Senders. Sie wurde von den Eigentümern der 49 Prozent der Aktien übernommen.

19) Formell war die Leitung des gesamtnationalen staatlichen Senders der Regierung der Russischen Föderation unterstellt. Allerdings verfügte die Regierung nicht über die Instrumente zur Leitung der Sendeanstalt. Nur der Präsident konnte über die Einstellung und Entlassung der Fernsehleitung entscheiden. Doch der Leiter von RTR konnte sich nicht direkt an den Präsidenten wenden, er wandte sich an die Präsidialverwaltung. So war der Leiter des staatlichen Senders direkt der Präsidialverwaltung unterstellt (Коломиец 2001, S. 88).

20) Der Sender erhielt vom Budget lediglich die Personalkosten, die restlichen Mittel musste er sich durch Werbeeinnahmen erwirtschaften. 
Die Kommerzialisierungsprozesse im Fernsehen wurden von den intensiven Prozessen der Innendifferenzierung begleitet. Es bildeten sich solche Teilbereiche innerhalb der Medienproduktion, wie 1) Nachrichten/Berichte, 2) Werbung und 3) Unterhaltung, heraus. Mit ihnen entstanden solche Typen der massenmedialen Organisationen wie Nachrichtenagenturen, Werbeagenturen und Organisationen, die Fernsehunterhaltung produzierten. Die Herausbildung der Fernsehmärkte sowie Kontakte zwischen neu entstandenen kommerziellen Sendern und internationalen Medienproduzenten stellten die begünstigenden Bedingungen für diese Prozesse der Innendifferenzierung dar. Denn die Zusammenarbeit mit westlichen Medienproduzenten ermöglichte die Nachahmung internationaler Routinen der Medienproduktion, was die Ingangsetzung der internationalen Diffusionsprozesse ${ }^{21}$ erlaubte.

(ad 1) So wurde das parteiliche Monopol auf die Sammlung und Verbreitung von Nachrichten bereits 1990 aufgehoben, als mit der Verabschiedung des Pressegesetzes die Auflösung des parteilichen Zensurapparats rechtlich zementiert wurde. Nach dem Zerfall der Sowjetunion, Ende 1991, wurde der »Gesetz über die Medien« verabschiedet, das die Ablehnung der Zensur bestätigte. In den 90er Jahren des letzten Jahrhunderts konnte das Monopol der staatlichen Nachrichtenagenturen TASS (1992 umbenannt in ITAR-TASS) und APN (1992 umbenannt in RIA-Vesti) sowohl auf der gesamtnationalen als auf der regionalen Ebene durchbrochen werden. Auf der gesamtnationalen Ebene konkurrierte mit den staatlichen bereits ab 1989 die erste kommerzielle Nachrichtenagentur Interfax ${ }^{22}$. Später, ab Mitte der 90er Jahre des 20. Jahrhunderts, gewannen die s.g. Weltnachrichtenagenturen (Reuters, Agence France-Presse und Associated Press) den Zugang zum Nachrichtenmarkt der Russischen Föderation, was den Konkurrenzdruck für die ITAR-TASS und RIA-Vesti erheblich erhöhte. Führende russische Nachrichtenagenturen mussten nun adäquate Nachrichtenprodukte anbieten, womit die Weltereignisse nicht mehr ignoriert werden konnten. Deshalb ist es hier angemessen, von der internationalen Diffusion der Fernsehnachrichten zu sprechen. Außerdem waren ab jetzt sowohl die staatlichen als auch die neuen privaten Fernsehsender in der Lage, zwischen unterschiedlichen Informationsanbietern auszuwählen. Zusammenfassend lässt sich festhalten, dass heute in Russland über 1000 Nachrichtenagenturen tätig sind (Засурсуий, Я. 2001, S. 210ff.). Obwohl die staatlichen Nachrichtenagenturen ITAR-TASS und RIA-Vesti (neben kommerzieller Agentur Interfax) auf dem Nachrichtenmarkt der Russischen Föderation dominieren, ist die Nachrichtenlandschaft durch die Pluralisierung gekennzeichnet. Bereits im Jahre 1994 existierten auf bestimmte Themen und Sparten orientierte Nachrichtenagenturen (z.B. Nachrichtenagenturen, die sich auf der Produktion der ökonomischen Nachrichten spezialisieren, wie »AEN« oder »Skeit-Press «), regionale Nachrichtenagenturen wurden gegründet (z.B. »NTR-Region«), und es entstanden Nachrichtenagenturen im Internet (z.B. »NSN - Nationalnaja sluschba novostej«). Mit anderen Worten konnte durch die Dezentralisierung der Nachrichtenquellen eine Pluralisierung der Themenausschnitte in den Medien erreicht werden.

(ad 2) Während die Herausbildung des massenmedialen Bereichs der Nachrichten durch die Entstehung der Nachrichtenmärkte begünstigt wurde, schaffte die Herausbildung der Fernsehwerbemärkte eine Voraussetzung für die Konstituierung des Werbebereichs im Fernsehen. Dabei fand die Konstituierung des Marktes für die Fernsehwerbung in zwei Phasen statt: In der ersten Phase (1991-1994) entstanden gleichzeitig der Fernsehwerbemarkt und der Markt für die Fernsehprogramme. Der Bedarf an qualitativ hochwertigen Unterhaltungs-

21) Zu internationalen Diffusionsprozessen in den Medien vgl. Stöber 2000.

22) Diese, später sehr erfolgreiche, Medienorganisation zeichnete sich durch die Orientierung an die westlichen Kriterien der Nachrichtenproduktion aus. Als Vorbildorganisation diente dabei die weltweit tätige Nachrichtenagentur Reuters. 
programmen (Talk-Shows, Fernsehserien, Filme) stieg, weil die Steigerung der Zuschaueraufmerksamkeit, die den Werbeauftraggebern verkauft werden konnte, zur zentralen Orientierung der Fernsehproduktion wurde. Im Zeitraum zwischen 1991 und 1994 waren die russischen Finanzunternehmen (z.B. MMM, Alisa, Ekarambus) die führenden Werbeauftraggeber im Fernsehen. In der zweiten Phase (ab 1995) dominierten die transnationalen Werbeauftraggeber (Procter\&Gamble, Uniliver, Wrigley, Stimorol, Nestle, Cadbury u.a) den russischen Fernsehwerbemarkt. Weil die russischen Werbeagenturen in dieser Phase sehr jung waren und keine Erfahrungen in der Zusammenarbeit mit den transnationalen Werbeauftraggebern hatten, beschränkte sich die Zusammenarbeit der transnationalen Konzerne auf die Niederlassungen der Internationalen Werbeagenturen (wie z.B. DMB\&B, BBDO, Ogilvy\&Mather, Young\&Rubicam usw.) in Russland. In der Regel wurden die Fernsehwerbespots der transnationalen Werbeauftraggeber ins Russische übersetzt, aber ansonsten unverändert ausgestrahlt. Diese Strategie führte zur Angleichung der Fernsehwerbespots im russischen Fernsehen mit der Fernsehwerbung westlicher Länder. Die Homogenisierung betraf sowohl die Strategien der Werbeproduktion als auch die Werbeinhalte (Ê̂ëîìeaäö 2001, S. 205). Kennzeichnend ist, dass mit der Einführung der Werbung in die Fernsehproduktion die oben erwähnte Trennung zwischen Staat und Fernsehen auf der Organisationsebene stabilisiert wird: Denn die kommerzielle Werbung stellte die alternative Finanzierungsquelle zum staatlichen Etat für die TV-Sender dar. Zugleich begünstigte die Werbung die Konstitution von kommerziell geprägten Entscheidungskriterien im Fernsehen. Obwohl der Staat nach wie vor den Einfluss auf die redaktionelle Linie der Sender auszuüben versuchte ${ }^{23}$, konnten sich die Sender auf diese Weise durch die zunehmende finanzielle Unabhängigkeit mehr und mehr von der staatlichen Bevormundung lösen.

(ad 3) Ähnlich wie der Werbebereich war auch der entstehende Unterhaltungsbereich durch internationale Diffusionsprozesse geprägt. Die Übertragung der internationalen Standarte der Unterhaltungsproduktion, wie z.B. Formen der Fernsehformate (Talk-shows, Quizshows), fand 1991-1993, wie oben erwähnt, durch den kostenlosen Zugang der neuen Fernsehsender zu den ausländischen Filmressourcen sowie durch den Kauf der Lizenzen für ausländische Filme und Serien (1994-1995) statt. Vor allem die entstehende Konkurrenz um die Zuschauer führte zur Zunahme der Unterhaltungsinhalte im Fernsehen. Während bis Ende der 80er Jahre des letzten Jahrhunderts fast ausschließlich sowjetische Filme im Fernsehen ausgestrahlt wurden, verringerte sich deren Anteil im Zeitraum von 1991 bi 1995 auf ein Drittel des gesamten Fernsehvolumens (36 Prozent). Amerikanische Fernsehfilme machten 32 Prozent des Filmumfangs aus. Die westeuropäischen Filme 15 Prozent $^{24}$. Der Gesamtumfang der Fernsehfilme betrug ca. 30 Prozent der Sendezeit (vgl. Коломиец 2001: 147). Der Bereich der Unterhaltung übernahm für das neue Fernsehen die Funktion der Sicherung der Zuschaueraufmerksamkeit (der Zuschauerquoten). Mit anderen Worten sicherte er die Anschlusskommunikation im entstehenden Mediensystem.

Diese Ausführungen machen deutlich, dass der Kommerzialisierungsprozess des Fernsehens mit der Dezentralisierung der medienpolitischen Strukturen einherging. Personaltransfer, Einwirkung auf die redaktionelle Linie und Monopolisierung des Medieneigentums sowie Zensur unterlagen der fortschreitenden Erosion. Zwar versuchte der Staat den neu gegründeten Sendern in Fällen regierungskritischer Berichterstattung - vor allem bei im Falle der kritischen Berichterstattung über den ersten Tschetschenienkrieg (1995-1995) - mit

23) So wurden regelmäßig die Leiter von Ostankion (ab 1994 ORT) und RTR ausgetauscht. 1992 wurde der Föderale Informationszentrum (FIZ) gegründet, der auf die redaktionelle Linie der Fernsehredaktionen einzuwirken hatte, 1993 wurde er jedoch aufgelöst. Ausführlicher zur Erosion der Mechanismen der Medienkontrolle vgl. Mickiewicz 1999, S. 109ff.

24) Die restlichen Prozente setzten sich aus den Fernsehfilmen anderer Länder zusammen. 
dem Lizenzentzug zu drohen, doch aufgrund der fortschreitenden Kommerzialisierung der Medien und aufgrund der Selbstbeschreibung der Journalisten als Vertreter der vierten Gewalt blieben diese Versuche wirkungslos. So ermöglichte in dieser Phase des Medienwandels die Kommerzialisierung des Fernsehens die Ausdifferenzierung eines Mediensystems, das sich am Prinzip der Aktualität orientiert, seine Veröffentlichungsentscheidungen durch kommerzielle geprägte Kriterien reguliert und ein Bild vom differenzierten Publikum pflegt.

\section{Entstehung eines informellen Bereichs innerhalb des Mediensystems (Ende 1995 - Anfang 2000)}

Die Medienlandschaft der Russischen Föderation zeichnete sich im Zeitraum zwischen Ende 1995 und Anfang 2000 durch Herausbildung eines neuen Typus der Medienkommunikation aus: Der informellen Public Relations. Neben einem autonomen Mediensystem, das sich auf der Grundlage des Prinzips der Aktualität und Neuheit reproduziert, entstand im neuen Russland eine Form der Medienkommunikation, die ihre Kriterien simulierte, zugleich aber durch informelle Aufträge zustande kam. So wurde es nach den Veränderungen der Medienstrukturen im Kontext der Präsidentenwahlen 1996 selbstverständlich, dass Fernseh- und Pressejournalisten Auftragsartikel und Auftragssendungen produzierten und veröffentlichten. Diese Entwicklung, die zum einen durch die Ko-Evolution der politischen und wirtschaftlichen Lenkungsstrategien des Fernsehens und zum anderen durch die Spaltung der postsowjetischen Übergangsgesellschaft in formelle und in informelle Strukturen begünstigt wurde, soll hier nun in Kürze nachgezeichnet werden.

Die Autonomisierung des Mediensystems zwischen 1991 und 1995 erlaubte nicht nur kritische Berichterstattung der Medien über den Tschetschenien-Krieg 1994-1995, sondern auch die Enttabuisierung solcher Themen wie »Gesundheit des Präsidenten« sowie »Präsident und Alkohol«, in denen Regierungsfähigkeit des amtierenden Präsidenten der RF Boris Jelzin von Medieninstanzen in Frage gestellt wurde. Zudem gewannen die oppositionellen Parteien die Parlamentswahlen von 1995 sehr erfolgreich, weshalb die Wahrscheinlichkeit der Wiederwahl des Präsidenten erheblich sank. Das für den sowjetischen Parteiapparat gängige Schema der Medienkontrolle erschien in diesem Kontext für die Regierung der Russischen Föderation als äußerst attraktiv. Doch, aufgrund der Kommerzialisierungsprozesse in den Medien, konnten die alten Kontrollmechanismen nicht mehr ohne weiteres eingesetzt werden. M.a.W. waren sowohl die (partielle) Zensur als auch die Einwirkung auf die redaktionelle Linie aufgrund der Herausbildung eines Mediensystems zu diesem Zeitpunkt nicht mehr wirksam. Aus diesem Grund sollten, nach Ansicht des Wahlstabs des Präsidenten, die Strategien der politischen PR nach westlichem Vorbild seinen Wahlerfolg sichern. Sie wurden jedoch, im Unterschied zu westlichen Ländern, zusammen mit klassischen Kontrollmechanismen des Fernsehens kombiniert.

So waren während der Wahlkampagne im Jahre 1996 monopolisierungsähnliche Prozesse im Fernsehen zu beobachten: Die führenden Medien, vor allem die Sender ORT und NTV, wurden in die so genannte »Koalition der Machtpartei« aufgenommen und sicherten die propräsidiale Berichterstattung. Auch der Mechanismus der Einstellung/Entlassung wurde eingesetzt: Der Leiter des staatlichen Senders RTR liberale, Oleg Popzov, wurde ausgewechselt. Schließlich wurden an diesen staatlichen Sender als auch in staatliche Radiostationen Anweisungen weitergeleitet, wie die Berichterstattung über die Präsidentenaktivitäten auszusehen hat. Gleichzeitig wurden die Methoden der Public Relations angewandt. In der Regel simuliert die PR die medialen Formen, um die Publikumsaufmerksamkeit zu erreichen. Auch der Wahlstab des Präsidenten spezialisierte sich auf Beobachtung der medialen Berichterstattung (Засурский. 2001, S. 66f.). Der Stab gliederte die wichtigsten Themen der medialen Agenda aus und versuchte die Präsidentenaktivitäten in die Nähe der zentralen Themen 
zu platzieren, was mit Hilfe der telefonischen Anweisungen an die Medien geschah, die in der »Koalition der Machtpartei« aufgenommen waren. Der Name des Präsidenten und seine Aktivitäten erschienen auf diese Weise im Kontext aktueller Berichterstattungen, ohne dass die Einwirkung auf die redaktionelle Linie sichtbar wurde. Die Aktivitäten der Opposition rückten in die mediale Peripherie. Die Wahlkampagne des Präsidenten wurde vom Erfolg gekrönt: 1996 wurde Boris Jelzin zum zweiten Mal zum Präsidenten der Russischen Föderation gewählt. Weil Public Relations hier mit Hilfe der informellen Mechanismen eingesetzt wurden, können wir diesen Typ der Medienkommunikation als die informellen PR bezeichnen.

Nach den Präsidentenwahlen wurden die oben beschriebenen staatlichen Lenkungsstrategien der Medien (Einwirkung auf die redaktionelle Linie, Einstellung/Entlassung, Teilmonopolisierung und informelle PR) sowohl von den politischen als auch von den wirtschaftlichen Organisationen übernommen. Zum einen lag es daran, dass die Regierung nach dem Wahlsieg des Präsidenten nach wie vor informelle PR als eine Form des Krisenmanagements einsetzte. Zum anderen war der Großteil der Journalisten und Medienmanagern, die in den landesweit führenden Fernseh- und Presseorganisationen tätig waren, in die Präsidentenwahlkampagne 1996 involviert worden, weshalb das Wissen um die Anwendungsmöglichkeiten der informellen PR nicht mehr der Regierung vorbehalten blieb. Im Kontext der heftigen Privatisierungskämpfe ab 1996 wurde dieses Wissen als höchst begehrenswert wahrgenommen. Einige wirtschaftliche Großorganisationen, die sich um die Privatisierung des attraktiven staatlichen Eigentums beworben haben, waren zugleich Inhaber der Fernsehund Pressemedien (Засурский, И. 2001, S. 255). Das neue Wissen um die Methoden der informellen PR wurde von ihnen zusammen mit der alten sowjetischen Formel übernommen, nach der die Kontrolle über die gesellschaftlichen Prozesse nur durch Medienkontrolle möglich ist. Der Medieneigentum erleichterte zudem die Anwendung der klassischen Kontrollmechanismen, wie Einwirkung auf die redaktionelle Linie (im Falle der Berichterstattung über Privatisierungsvorgänge), Personaltransfer (im Falle der Wechsels der Hauptredakteure) sowie Teilmonopolisierung der Medienorganisationen (durch Bildung der Medienkonzer$\mathrm{ne}^{25}$ ). Auf diese Weise fand die Verbreitung der Methoden der informellen PR (Auftragsartikel und Auftragssendungen, Auftragsnachrichten) und die Wideraktualisierung klassischer sowjetischer Kontrollmechanismen nicht nur im Zentrum sondern auch in den Regionen Russlands statt.

Auch die fehlende journalistische Professionalität war für die Entstehung der informellen PR innerhalb der medialen Produktionsstätten mitursächlich (Кузнецов 2002; Лазутина $2001)^{26}$, denn russische Journalisten hatten die Anpassungsstrategie - Anpassung an die Obrigkeit, ob Partei, Staat oder Auftraggeber -, die sie in der Sowjetunion gepflegt haben, beibehalten. Selbst die Verfechter der Idee der vierten Gewalt mussten eingestehen, dass die russischen Journalisten eher dazu neigen, die Anweisung des Auftraggebers zu erfüllen als die redaktionelle Linie zu verteidigen. Zudem stellten die informellen PR - einen Auftragsartikel zu veröffentlichen oder auf bestimmte Personen zugeschnittene Sendung zu produzieren - eine lukrative Einkommensquelle für viele Journalisten dar. Folglich haben sich informelle Regeln der Auftragsartikel herausgebildet. Wie der Auszug aus dem Interview (2001) illustriert, verfestigten sich in den (Fernseh-)Redaktionen in diesem Zeitraum nicht die professionellen Standarte, sondern die Standarte der »Käuflichkeit« eines Journalisten:

25) Zur Übersicht über die staatlichen und privaten Medienkonzerne im Zeitraum 1995-1999 vgl. 3acypский 2001, S. 255f.

26) Zusätzlich wurden die Verbreitung der informellen Strukturen und steigende Anpassungstendenzen in der journalistischen Gemeinschaft durch die Krise des Fernsehwerbemarktes in den Jahren 19971998 begünstigt. Die letzte war wiederum durch die Währungskrise verursacht worden. 
»Es gibt bestimmte Spielregeln, wenn man von einem Menschen etwas erhält... Wenn Journalisten Zeitungen lesen und fernsehen, rechnen sie sofort die Auftragsartikel aus und benennen die Preise. Zeitweise sagt man: >Schau mal, er war bei uns und wir haben ihn weggeschickt, weil er zu wenig Geld brachte, aber die anderen haben seinen Auftrag angenommen, haben den Preis gedrückt.<...Es gibt einfach bestimmte Regeln: Wenn man den Auftrag eines Menschen erfüllt, seinen beauftragten Artikel oder Sendung veröffentlicht, aber er kein Geld zahlt, wird dies sofort über ein >Telefon< weitergegeben und über diesen Menschen wird nirgendwo mehr berichtet. Oder wenn es mit einem Kunden Probleme gibt, wird dies in der Regel über eigene nicht-öffentliche Kanäle weitergegeben. Und wenn alles in Ordnung ist, wird dies auch weitergegeben, dass man diesen Kerl senden darf. In unseren Medien herrscht heute der Kodex der Finanzehre, sozusagen.« (Auszug aus dem Interview Nr. 18, Übersetzung d. Verfasserin, Quelle: eigene Erhebung 2001)

Die Entstehung des informellen PR-Bereichs in Fernsehorganisationen und anderen Medien ist auf die Spaltung zwischen formeller und informeller Ebene in der russischen Übergangsgesellschaft zurückzuführen. Da sich gesellschaftliche Institutionen im neuen Russland im intensiven Wandlungsprozess befinden, übernehmen informelle Strukturen die Funktion der Verarbeitung politischer und wirtschaftlicher Konkurrenzprobleme in der Übergangsphase. Diese informellen Strukturen innerhalb der Medien irritierten die journalistische Autonomie. Aber die Irritationen verliefen dezentral, weil die Ko-Evolution politischer und wirtschaftlicher Lenkungsstrategien der Medien die Situation schuf, in der Redaktionen und Medien nicht unter dem Einfluss einer Auftraggeber-Organisation, sondern unter dem Einfluss mehrerer, untereinander konkurrierender, Auftraggeber-Organisationen standen.

\section{Evolution staatlicher Kontrollmechanismen des Fernsehens (2000-2006)}

Ab 2000 lassen sich im neuen Russland die Zentralisierungstendenzen der staatlichen Medienkontrolle beobachten. In dieser Phase versuchte der Staat eine hierarchische Unterordnung des Fernsehens zu erreichen. Das Mediensystem wurde durch die Zunahme der informellen staatlichen Kontrolleingriffe gestört. Auch die thematische Offenheit der Nachrichteninhalte wurde in Bezug auf die Thematisierung der Regierung des Präsidenten partiell eingeschränkt. Zugleich blieb der Bereich der informellen PR-Kommunikation innerhalb der (Fernseh-) Redaktionen nach wie vor bestehen. Hier wird ein kurzer Überblick dieser Entwicklungen präsentiert.

Nach der Wahl von Vladimir Putin zum Präsidenten der Russischen Föderation im März 2000 hat sich die staatliche Medienpolitik verändert. Wenn zwischen 1995 und 2000 die Regierung zumindest offiziell Freiheit der Presse tolerierte, wurde ab 2000 in Russland die »Politik der gelenkten Demokratie« eingeführt, in der den Medien eine besondere Rolle zugedacht war. Das offizielle Ziel dieser Politik war die Durchführung der politischen und rechtlichen Reformen in Russischer Föderation. Allerdings wurde die Zentralisierung der politischen Macht beim Präsidenten und seinem Apparat als notwendig postuliert ${ }^{27}$, um weitere demokratische Standarte und rechtliche Normen in Russland zu verfestigen. Die Notwendigkeit der Machtzentralisierung wurde dabei mit dem Übergangscharakter rechtlicher und politischer Institutionen in RF begründet: Da sich die entsprechenden Institutionen noch nicht vollständig herausgebildet hatten und nach wie vor Fehlentwicklungen aufwiesen, war eine übergeordnete Instanz notwendig, die die Entwicklungsrichtung überwachte - der Präsident und seine Verwaltung. Deshalb wurden die Abwesenheit von Opposition im Parlament sowie die telefonischen Anweisungen der Regierungsbeamten an die Gerichte nicht als undemokratisch oder rechtswidrig angesehen. Die Bevölkerung wurde aus dieser Perspektive als >Masse' wahrgenommen, der die >richtige' Demokratie vermittelt werden sollte. Diese Auf-

27) Diese Politik fand auch in der Veränderung der Sitzordnung im Sowjet der Föderation (der Oberen Kammer des Parlaments) ihren Ausdruck. Diese Reform (2001) bestand vor allem darin, dass nicht mehr die Gouverneure, sondern ihre Vertreter in der Oberen Kammer des Parlaments tagten. 
gabe sollten nun die Medien übernehmen. So gewann die Idee der staatlichen Kontrolle der Medien in dieser Phase wieder an Attraktivität. Wenn die Regierung in der früheren Phase eher zu den Techniken der informellen PR griff, wurde ab 2000 die Tendenz zur Nutzung >klassischer' Kontrollmechanismen der Medien verstärkt. Zu diesen Mechanismen zählen in erster Linie 1) Der Mechanismus der Teilmonopolisierung und 2) der Mechanismus der Einwirkung auf die redaktionelle Linie.

(ad 1) Der Prozess der (Teil-)Monopolisierung begann mit informeller Erpressung der Medienorganisationen durch den Staat. Dabei dienten formelle Fehler, z.B. Nicht-Erfüllung bestimmter gesetzlicher Regelungen, die im Zweifelsfalle unternehmensschädigend ausgelegt werden können, als Vorwand. So wurden im Jahre 2000 als erstes die Ausstrahlungslizenzen zweier Fernsehsender ORT und TVZ durch das Presseministerium zur Neuvergabe ausgeschrieben. Nach dem Bewerbungsverfahren durften die Sender ihre Lizenzen beibehalten. Aber das Verfahren war die öffentliche Vorführung, wie kritische Äußerungen durch staatliche Instanzen sanktioniert werden können. Dabei dienten dem Staat formelle Fehler beider Sender als Anlass, um die Freigabe der Lizenzen zu initiieren ${ }^{28}$.

Als nächste Phase der Monopolisierung ist die Auflösung des Journalistenteams sowie der Eigentümerwechsel des kommerziellen Senders NTV (2000-2001) zu nennen. Der Sender musste von seinem Eigentümer und Medienmogul Vladimir Gussinski ${ }^{29}$ an den staatsnahen Konzern Gazprom verkauft werden, der heute zu 51 Prozent dem Staat gehört. Kurz danach fand ein ähnlicher Vorgang mit dem kommerziellen Sender TV-6 statt (dessen Miteigentïmer der im Exil lebende Medienmogul Boris Beresovski war), zu dem das Journalistenteam von NTV gewechselt hatte (Mitte 2001 bis Anfang 2002). Schließlich beschloss das Journalistenteam, das zuvor von NTV auf TV-6 wechselte und mit Schließung von TV-6 gehen musste, einen eigenen Sender, TVS, zu gründen. Führende öl- und metallverarbeitende Unternehmen haben die Finanzierung des Senders übernommen. Im Laufe eines Jahre (20022003) musste der neue Sender TVS jedoch schließen, weil seine Arbeit aus komplizierten rechtlichen Gründen nicht fortgesetzt werden konnte. Es ist nicht unwahrscheinlich, dass auch in diesem Fall die Monopolisierungsstrategie in Gang gesetzt wurde. Denn bereits ab Juni 2003 sendete auf der Frequenz des ehemaligen Senders TV-6 und TVS nun der staatliche Sender Sport, dessen thematische Ausrichtung keine kritische Berichterstattung über Regierungspolitik zuließ.

(ad 2) Die Anwendung des Mechanismus der (Teil-)Monopolisierung durch den Staat geht mit der Einwirkung auf die redaktionelle Linie der ausgewählten Fernsehsender einher. Dieser Mechanismus wird vor allem in den staatlich kontrollierten Fernsehsendern ORT ${ }^{30}$, RTR $^{31}$, NTV (kontrolliert durch Gazprom) und TVZ angewandt. Dies bestätigt sich zum einen durch die Aussagen von Fernsehjournalisten in den von mir durchgeführten Interviews (2001) und zum anderen durch die Interviews mit russischen Fernsehjournalisten aus anderen Quellen ${ }^{32}$. Die Leiter der Fernsehsender erhalten die Vorgaben über die Berichterstattung von Präsidenten- und Regierungsaktivitäten durch die Mitarbeiter des Präsidentenam-

28) Außerdem wurde Boris Beresovski im Jahre 2000 der Einfluss auf die redaktionelle Politik des führenden Senders Russlands, ORT, genommen. Auf Empfehlung des Kremls reiste der Milliardär aus Russland aus. Alluminium- und Ölkonzerne sind formell Teilhaber von ORT. Informell übernahm die Präsidialverwaltung den Einfluss auf die redaktionelle Linie dieses Senders.

29) Der Medienmogul musste den Vertrag über den Verkauf des Senders im Gefängnis unterzeichnen, wo er einige Wochen festgehalten wurde. Bemerkenswert ist, dass bei der Übergabe des Vertrags der Presseminister der RF und der Leiter von Gazprom anwesend waren.

30) ORT wurde 2002 in Pervij Kanal umbenannt.

31) RTR wurde 2002 in Rossija umbenannt.

32) Vgl. Журнал »Отечественные записки»« (2003), in: http://www.strana-oz.ru (April 2004). 
tes. Von Fernsehleitern werden diese Vorgaben an die Chefredakteure weitergegeben. Sie wiederum verbreiten diese Anweisungen an die Journalisten ${ }^{33}$. Einige Interviewausschnitte illustrieren diese These:

»[Frage der Verfasserin]: Wer schränkt [Inhalte] ein, wer filtert die Information?

[Antwort eines Fernsehjournalisten]: All das geschieht nicht direkt. Beresovski zahlt nicht direkt, der Staat kontrolliert nicht direkt. Wenn wir von großen gesamtnationalen Fernsehsendern sprechen, so wie sie vor paar Jahren gewesen sind - betrachten wir die Gesamtsituation: ORT, RTR, NTV - da existierte eine mehrstufige Struktur. Der Staat kontrolliert [uns] nicht direkt, [uns] kontrollieren Menschen, die einmal wöchentlich in den Kreml fahren, die Meinung des Staates erfahren, oder sie fahren einmal wöchentlich, so wie das mit ORT gewesen ist, zu Beresovski, und erfahren seine Meinung. Dann - ein Mix - das ist das, was wir auf dem Bildschirm sehen. [...] Oligarchen gibt es heute nicht mehr, und es ist offensichtlich - es werden schwerpunktmäßig staatliche Ansichten vermittelt.« (Auszug aus dem Interview Nr. 31, Übersetzung d. Verfasserin, Quelle: eigene Erhebung 2001).

Interessant ist, dass diese Vorgaben auf verschiedene Weise an die Fernsehleitung gelangen können. Zum einen existieren so genannte »Pressemanager« (häufig die Mitarbeiter der Pressestellen von Regierungsinstanzen), die die Anweisungen persönlich übermitteln und die Koordination der Absender weitergegeben werden, falls diese durch mehrere Eigentümer übermittelt werden (s. oben $)^{34}$ Zum anderen können diese Anweisungen telefonisch an die Fernsehleitung weitergereicht werden ${ }^{35}$ :

»[I]ch glaube, dass es kein Zufall ist, dass auf Arbeitstischen der Leiter großer Fernsehsender nach wie vor > vertuschki< stehen, so wie es früher war. [...] Wissen Sie, was das ist? Das ist direkte Verbindung zu Kreml, zur Präsidialverwaltung. Und sie funktioniert, verstehen Sie?! So wie die früher standen. Ich war vor kurzem, gerade vor einem Monat, im Arbeitszimmer des Fernsehleiters eines der gesamtnationalen Fernsehsender. Auch bei ihm steht >vertuschka<, so wie sie bei seinen Vorgängern vor zehn Jahren stand.« (Auszug aus dem Interview Nr. 8, Übersetzung d. Verfasserin; Quelle: eigene Erhebung 2001)

Während die staatlichen und staatsnahen Sender, wie NTV, mit der Einwirkung auf die redaktionelle Linie konfrontiert werden, werden die nicht-staatlichen Sender durch Behinderung des Informationszugangs beeinflusst. Die Untersuchung einer angesehenen russischen Stiftung von 2003, in der 1370 Journalisten befragt wurden, bestätigt, dass vor allem nichtstaatliche Medien von Nicht-Akkreditierung und von anderen Formen der Informationsbehinderung, wie unbegründete Behinderung des Zugangs in die Räume, unbegründete Behinderung der Audio- und Video-Aufnahmen, unbegründete Absage der Herausgabe von Daten, durch staatliche Ämter betroffen sind ${ }^{36}$. Die Absage der Informationsausgabe wird häufig mit dem geheimen Charakter der Information und mit dem Verbot der Vorgesetzten begründet.

Schließlich ist diese Phase durch die Wideraktualisierung der journalistischen Selbstzensur sowohl in den staatlichen als auch in den privaten Sendern gekennzeichnet. Das Vorhandensein dieses Mechanismus erklärt, warum sich trotz Pluralisierung der Medienorganisationen journalistische Selbstkontrolle einsetzt ${ }^{37}$. Zum anderen weist die Wideraktualisierung der Selbstzensur darauf hin, dass die Strukturen des bestehenden Mediensystems durch Zentralisierung der staatlichen Medienkontrolle doch in ihrer Eigenlogik partiell und temporär eingeschränkt werden (Шендорович 2004).

33) Vgl. auch Interview Nr. 2, Nr. 6, Nr. 24, Nr. 28, Nr. 29, Nr. 32, Quelle: eigene Erhebung 2001.

34) Vgl. auch Interview Nr. 29, Nr. 31 (2001), Quelle: Eigene Erhebung.

35) Vgl. auch Interview Nr. 12, Nr. 24 (2001), Quelle: Eigene Erhebung.

36) Siehe Untersuchung »Общественная экспертиза« (2003) der Stiftung »Фонд защиты гласности«, in: http://www.gdf.ru (Januar, 2005).

37) Seit 2003 existierten in der gesamten Russischen Föderation neben 9 führenden überregionalen Sendern über 1600 regionale, sowohl staatliche als auch private Fernsehsender (Шариков 2003a und 2003b). 


\section{V.}

Die Darstellung der Fernsehstrukturen zwischen 1970 und 2006 hat gezeigt, dass modernisierungstheoretische Beschreibungskonzepte des Medienwandels - nach dem Muster »von Propaganda zu autonomen Medien« - den komplexen Verlauf der medialen Veränderungen nicht erklären können. Stattdessen wird die Einmaligkeit und regionale Spezifität der medialen Veränderungsprozesse sichtbar. Diese Spezifität kann aus einer differenzierungstheoretischer Perspektive als Konditionierung der sozialen Strukturen durch den komplexen Übergangskontext verstanden werden. So bestimmen die charakteristischen Merkmale beider gesellschaftlichen Differentzierungsformen - Organisationsgesellschaft (Zentralisierung, Geschlossenheit und Trennung zwischen formellen und informellen Strukturen) und der funktionalen Differenzierung (funktionale Differenzierung, Offenheit, Minimierung der Trennung zwischen formellen und informellen Strukturen) - den Verlauf aber auch die Widersprüchlichkeit der postsowjetischen Transformationspfade.

Der oben beschriebene Medienwandel kann somit als Pendeln zwischen diesen antinomischen Eigenschaften charakterisiert werden. So finden die zentralen Merkmale der Organisationsgesellschaft hervorragend ihren Ausdruck in den Propagandamedien (1970-1985). Das Merkmal der Zentralisierung lässt sich in der Unterordnung des Fernsehens und anderer Medien unter den Parteiapparat ablesen. Auch die inhaltliche Geschlossenheit der Propagandamedien sowie die strukturelle Trennung in einen formellen und einen informellen Medienbereich sind beobachtbar. Mit dem Beginn des Medienwandels (1986-1991) ist zwar die hierarchische Unterordnung der Medien dem Parteiapparat noch gegeben (M.a.W. existiert das Zentralisierungsmerkmal fort), doch lässt sich bereits eine thematische Öffnung sowie Legalisierung der informellen Medien feststellen. Bereits in der zweiten Phase des Medienwandels (1991-Ende 1995) können Medien autonom auf der Organisationsebene Entscheidungen produzieren, was auf den entscheidenden Vorgang der Dezentralisierung und einer funktionalen Ausdifferenzierung des Mediensystems hindeutet. Aber auch die thematische Offenheit der Nachrichten- und Unterhaltungsbereiche weitet sich aus, während die Trennung zwischen formellen und informellen Strukturen innerhalb der Medien durch den Kommerzialisierungsprozess kaum von Relevanz ist. In der dritten Phase des Wandels (Ende 1995 - Anfang 2000) lassen sich einzelne Versuche einer hierarchischen Unterordnung des Fernsehens feststellen. Weil diese eher dezentral verlaufen, kann hier vom Pendeln zwischen Zentralisierung und funktionaler Differenzierung gesprochen werden. Folglich wächst die Trennung zwischen der formellen und der informellen Ebene innerhalb der Medien. Die thematische Offenheit bleibt jedoch bestehen. Schließlich kann die (vorläufig) letzte Phase des Medienwandels (ab Anfang 2000) als Schwingen zwischen funktionaler Differenzierung und gesellschaftlicher Zentralisierung charakterisiert werden, da hierarchische Unterordnung des Fernsehens unter Regierungsinstanzen aufgrund der Änderung der Eigentümerverhältnisse (insbesondere im Fernsehen) zunimmt. Gleichzeitig besteht die Trennung zwischen formellen und informellen Medienstrukturen fort, während die thematische Offenheit der Medien in Nachrichten partiell eingeschränkt wird. Somit kann hier die abschließende These des Artikels formuliert werden, dass das entstandene eigenlogische Mediensystem im heutigen Russland über keine Exklusivität auf die Leistung der Informationsproduktion verfügt. Neben ihm leisten im postsowjetischen Gesellschaftsraum die informellen Medienstrukturen sowie Elemente der Propaganda eine machtvolle Schatten-Produktion von gesellschaftlichen Realitäten. 


\section{Literatur:}

Androunas, Elena (1993): Soviet Media in Transition. Structural and Economic Alternatives, Westport.

Blöbaum, Bernd (1994): Journalismus als soziales System. Geschichte, Ausdifferenzierung und Verselbstständigung, Opladen.

Eisenstadt, Shmuel N. ([1980], 1982): Vergleichende Analyse der Staatenbildung in historischen Kontexten, in: Stefan Breuer/Hubert Treiber (Hrsg.), Entstehung und Strukturwandel des modernen Staates, Opladen, S. 36-74.

Eisenstadt, Shmuel N. (1990): Functional Analysis in Anthropology and Sociology. An interpretative Essay, in: Annual Review of Anthropology 19, S. 243-260.

Eisenstadt, Shmuel N. (Hrsg.) (1992): Kulturen der Achsenzeit II. Ihre institutionelle und kulturelle Dynamik, 3 Bände, Frankfurt am Main.

Eisenstadt, Shmuel N. (1999): Die Vielfalt der Moderne, Weilerswist.

Ettrich, Frank (1992): Von der Erfolgswissenschaft zur Krisenwissenschaft. Die gesellschaftlichen Transformationsprozesse in Osteuropa und Ostdeutschland als Herausforderung der deutschen Soziologie, in: Hansgünter Meyer (Hrsg.), Soziologie in Deutschland und die Transformation großer gesellschaftlicher Systeme. Diskussionsreden des Plenums und der 38 Arbeitsgruppen und Foren des Soziologentages und der 7. Tagung für angewandte Soziologie des Berufsverbandes Deutscher Soziologen in Leipzig vom 24.-26. Mai 1991, Berlin, S. 122-141.

Lasswell, Harold (1927): Propaganda technique in the World War, London, New York.

Lasswell, Harold (1995): Propaganda, in: Robert Jackhall (Hrsg.): Propaganda, London, S. 13-25 (zuerst abgedruckt in: Encyclopedia of the Social Sciences. Bd. 12, London, 1934).

Luhmann, Niklas (1975): Weltgesellschaft, in: Soziologische Aufklärung 2, 3. Aufl., Wiesbaden, S. 51-71.

Luhmann, Niklas (1996): Realität der Massenmedien, 2. Aufl., Opladen.

Luhmann, Niklas (1998): Die Gesellschaft der Gesellschaft, 1.Aufl., Frankfurt am Main.

Marcinkowski, Frank (1993): Publizistik als autopoietisches System. Politik und Massenmedien. Eine systemtheoretische Analyse, Opladen.

Merten, Klaus (2000): Struktur und Funktion der Propaganda, in: Publizistik. Vierteiljahreshefte für Kommunikationsforschung, Jg. 45, Heft 2, S. 143-162.

Mickiewicz, Ellen (1999): Changing Channels. Television and the Struggle for Power in Russia, Durham and London.

Müller, Monika (2001): Zwischen Zensur und Zäsur. Das sowjetische Fernsehen unter Gorbatschow, Wiesbaden.

Murray, John (1994): The Russian Press from Brezhnev to Yeltsin. Behind the Paper Curtain, Aldershot.

Pollack, Detlef (1994): Die Kirche in der Organisationsgesellschaft. Zum Wandel der gesellschaftlichen Lage der evangelischen Kirchen in der DDR, Stuttgart.

Sinowjew, Alexandr (1981): Kommunismus als Realität, Zürich.

Stichweh, Rudolf (2000): Die Weltgesellschaft. Frankfurt am Main: Suhrkamp.

Stöber, Rudolf (2000): Die Adaption und Diffusion von Medienkonzepten als Motor der Ausdifferenzierung des Mediensystems, in: Hans-Bernd Brosius (Hrsg.), Kommunikation über Grenzen und Kulturen, S. 59-75.

Thomas, Barbara/Tzankoff, Michaela (2001): Medien und Transformation in Osteuropa, Wiesbaden

Voltmer, Klaus (2000): Massenmedien und demokratische Transformation in Osteuropa. Struktur und Dynamik öffentlicher Kommunikation im Prozeß des Regimewechsels, in: Hans-Dieter Klingemann/ Frank Neidhardt (Hrsg.), Zur Zukunft der Demokratie. Herausforderungen im Zeitalter der Globalisierung, Berlin, S. 123-152.

Weischenberg, Siegfried (1994): Journalismus als soziales System, in: Klaus Merten/Siegfried Schmidt/ Siegfried Weischenberg, (Hrsg.), Die Wirklichkeit der Medien. Eine Einführung in die Kommunikationswissenschaft, Opladen, S. 427-454. 
Zapf, Wolfgang (1990): Modernisierung und Modernisierungstheorien, in: Wolfgang Zapf (Hrsg.), Die Modernisierung moderner Gesellschaften. Verhandlungen des 25. Deutschen Soziologentages in Frankfurt am Main 1990, Frankfurt am Main, S. 23-39.

Zapf, Wolfgang (1994): Modernisierung, Wohlfahrtsentwicklung und Transformation. Soziologische Aufsätze 1987 bis 1994, Berlin.

\section{Russischsprachige Quellen:}

Грабельников, Александр (2001): Массовая информация в России. От первой газеты до информационного общества, Москва

Журнал „Отечественные записки“ (2003), in: http://www.strana-oz.ru (März 2003).

Засурский, Иван (2001): Реконструкция России. Масс-медиа и политика в 90-е, Москва.

Засурский, Ясен (Hrsg.) (2001): Система средств массовой информации России, Москва.

Коломиец, Виктор (Hrsg.) (2001): Телерекламный бизнес, Москва.

Кольцова, Елена (1999): Производство новостей: скрытые механизмы контроля, in: Журнал. социологии и социальноя антропологии, Том II, № 3.

Кольцова, Елена (2001): Производство новостей. Типы „Влиний“ на работу журналистов, in: Невидимые грани социальной реальности, Санкт-Петербург, S. 109-120.

Кордонский, Симон (2000): Рынки власти. Административные рынки СССР и России, Москва.

Кузнецов, Георгий (2002): ТВ-Журналистика. Критерии профессионализма, Москва.

Кузьмич, Валерий/Прашкович, Артур, (2001): Секреты российского политического PR.

Лазутина, Галина (2001): Профессиональна этика журналиста, Москва.

Левада, Юрий (2003): Рамки и варианты исторического выбора: несколько соображений о ходе российской трансформации, Москва.

Ленин, Владимир (1958): Ленин в печати, Москва.

Муратов, Сергей (2001): Эволюция нетерпимости. История и конфликты этических представлений, Москва.

Прайс, Монро (2000): Телевидение, телекоммуникации и переходный период. Право, общество и национальная идентичность, Москва

Федотов, Михаил (2002): Право массовой информации в Российской Федерации, Москва.

„Фонд защиты гласности“ (2003): „Общественная экспертиза“, in: http:/www.gdf.ru (Januar 2005).

Шариков, Александр (2003a): 10000 километров эфира, in: Журнал „Индустрия рекламы“ № 23 , S. $20-23$.

Шариков, Александр (2003b): Телевизионное и радиовещание, in: Новая российская энциклопедия, Москва, S. 651-654.

Шендерович, Виктор (2004): Цензура сегодня дело добровольное, in: Журнал „Индекс“ 20/2004,in: www.index.org.ru (Mai 2005).

Цвик, Валерий/Назарова, Яна (2002): Телевизионные новости Россиии, Москва.

Dr. Anna Amelina

Universität Bielefeld

Fakultät für Soziologie

Postfach 33501, Bielefeld

e-mail: anna.amelina@uni-bielefeld.de 